\title{
TRIANGULAÇÃO EM ESTUDOS DE CASO: INCIDÊNCIA, APROPRIAÇÕES E MAL-ENTENDIDOS EM PESQUISAS DA ÁREA DE ADMINISTRAÇÃO
}

\author{
TRIANGULATION IN CASE STUDIES: INCIDENCE, APPROPRIATIONS AND
}

MISUNDERSTANDINGS IN MANAGEMENT STUDIES

Recebido em: 08/12/2017 • Aprovado em: 19/02/2018 Avaliado pelo sistema double blind review Editor Científico: Edson Sadao Iizuka DOI 10.13058/raep.2018.v19n2.889

\section{CAMILA BRUNING camila.bruning@gmail.com \\ Universidade Federal do Paraná}

\section{LUCIANA GODRI}

Pontifícia Universidade Católica do Paraná

\section{ADRIANA ROSELI WÜNSCH TAKAHASHI}

\author{
Universidade Federal do Paraná
}

RESUMO

Este artigo apresenta uma análise bibliométrica em que se identificou a incidência do uso e apropriações da triangulação como estratégia metodológica em Estudos de Caso publicados em periódicos científicos da área de Administração no Brasil. Entende-se, aqui, a triangulação como estratégia importante para aumentar a compreensão em profundidade dos fenômenos estudados, bem como atribuir maior confiabilidade e fidedignidade aos Estudos de Caso, nesse sentido, o estudo centrou-se em cinco modalidades de triangulação: (i) triangulação de fontes de evidência, (ii) de métodos, (iii) de investigadores, (iv) teórica, e (v) a técnica de triangulação tal como proposta por Triviños (1987). A pesquisa analisou uma amostra de 1.384 Estudos de Caso publicados, entre os anos de 2008 e 2012, em periódicos da área classificados como A1 até B1 pela Coordenação de Aperfeiçoamento de Pessoal de Nível Superior (CAPES) (conforme evento de qualificação Qualis 2013). Os resultados permitiram concluir que a triangulação é pouco utilizada em Estudos de Caso na área de Administração nacional (é relatada em apenas $12 \%$ dos estudos analisados). Nos poucos casos em que é utilizada, os tipos de triangulação privilegiados são fontes de evidência (100\%) e método $(86,72 \%)$, sendo raros estudos de caso que relatam a realização de triangulação teórica, de investigadores, ou técnicas de triangulação sistematizadas, como a proposta por Triviños (1987). Além da incidência e da apropriação que se faz da triangulação, foram identificados diversos equívocos no relato da utilização de triangulação. O texto explora esses mal-entendidos e apresenta sugestões para garantir o correto uso e relato da triangulação. Justifica-se a relevância do artigo dada a necessidade de refletir sobre a qualidade dos procedimentos metodológicos e dos relatos de pesquisa que se tem realizado na área de Administração.

Palavras-chave: Metodologia de pesquisa qualitativa em Administração. Estudos de caso. Triangulação.

\footnotetext{
ABSTRACT

This paper presents a bibliometric analysis that identifies the incidence of the use of triangulation, as well as its appropriations as a methodological strategy. It analyses case studies published in Brazilian scientific journals in the academic field of Management. Triangulation is understood as an important strategy to increase the in-depth understanding of the phenomena studied, as well as to assign greater reliability and trustworthiness to Case Studies. This work discuss five types of triangulation: (i) of sources of evidence; (ii) of methods, (iii) of researchers, (iv) of theories, and ( $v$ ) the triangulation technique as proposed by Triviños (1987). This research analyzed a sample of 1384 Case Studies published between 2008 and 2012 in periodicals classified as A1 to B1 by the Brazilian Coordination of Improvement of High-Education Personnel - CAPES (according to the 2013 Qualis qualification event). The results point out that only $12 \%$ of the studies analyzed reported the use of triangulation. In the few cases in which it was used, the studies reported triangulation of sources of evidence (100\%), triangulation of methods (86.72\%), with rare case studies reporting having used theoretical triangulation, or any type of systematized triangulation techniques, as the one proposed by Triviños (1987). In addition to the incidence and appropriation of triangulation, different misunderstandings have been identified regarding triangulation. This article explores these misunderstandings and presents suggestions to ensure the correct use and reporting of the technique. The relevance of the article is based on the need to reflect about the quality of the methodological procedures and of the research reports that have been carried out in the field of Business Administration in Brazil. Keywords: Qualitative methodology in Business Administration studies. Case studies. Triangulation.
} 


\section{INTRODUÇÃO}

Um dos instrumentos, frequentemente, citados na literatura de pesquisa qualitativa como uma estratégia metodológica para atribuir validade e fidedignidade aos estudos de caso é a triangulação. Nesse sentido, destacam-se os trabalhos de Eisenhardt (1989), Merriam (1998), Creswell (2007), Denzin, Lincoln e Netz (2007), Angrosino (2009), Flick (2009), Yin (2010; 2011), Bluhm et al. (2011), bem como o de Coraiola et al. (2013).

De acordo com a revisão de literatura apresentada neste artigo, a triangulação é trabalhada de diferentes maneiras pelos diversos autores, relacionando-a desde a:

(i) triangulação de fontes de evidência;

(ii) triangulação de métodos de coleta e análise de dados;

(iii) triangulação de teorias;

(iv) triangulação de pesquisadores.

É possível ainda identificar, na literatura sobre metodologia, autores que propõem técnicas de triangulação como um processo sistematizado e indicam um conjunto de procedimentos metodológicos específico, conforme um modelo de passo a passo a ser seguido, como é o caso da (v) técnica de triangulação proposta por Triviños (1987).

O objetivo deste artigo foi analisar como a área acadêmica de Administração brasileira tem adotado a triangulação em seus procedimentos metodológicos, e, mais além, que uso tem feito das diversas formas de triangulação supraelencadas.

Para isso, foram analisados artigos publicados em periódicos classificados como estrato A1 até B1, conforme o evento de classificação Qualis 2013, que se declaram como estudos de caso e relatam que utilizaram triangulação, então, identificando quais as formas e o tratamento de triangulação utilizados nestes trabalhos. A escolha por publicações nacionais recentes da área de Administração que adotaram o estudo de caso se deu por defender-se, conforme Coraiola et al. (2013), que este é o delineamento de pesquisa qualitativa mais utilizado na academia de Administração brasileira. 
A análise que aqui se apresenta concentrou seu foco nas seguintes formas de triangulação, como citadas anteriormente: (i) A triangulação de fontes de evidência; (ii) triangulação de métodos de coleta e/ou análise de dados; (iii) triangulação de teorias; (iv) triangulação de pesquisadores; e também (v) técnica de triangulação proposta por Triviños (1987).

A técnica de triangulação proposta por Triviños (1987) foi escolhida, como exemplo, dentre diversas outras formas sistematizadas de triangulação, porque apresenta uma sequência de passos metodológicos específicos e característicos, o que indica o uso de um autor em particular - mesmo que ele não seja diretamente citado.

Os conceitos de triangulação são diversos para diferentes autores, variando a partir das suas abordagens epistemológicas e podendo assumir desde uma forma mais positivista, como no caso da proposta de Yin (2010), até formas mais qualitativas, como na proposta de Eisenhardt (1989). O intuito deste trabalho não foi discutir as contradições, os questionamentos e as melhores indicações para casos específicos, e sim, assumir que estudos de caso em Administração, realizados segundo as mais diversas tradições epistemológicas, podem ter ganhos de qualidade ao utilizar as diversas formas de triangulação como estratégia metodológica para aumentar sua fidedignidade e validade de pesquisa.

Este artigo está estruturado da seguinte maneira:

A seguir é apresentada uma seção de embasamento teórico na qual se explana sobre os entendimentos, diferenciais e tipos de triangulação, conforme indicado por diferentes autores, tais como Triviños (1987), Eisenhardt (1989), Merriam (1998), Creswell (2007), Denzin, Lincoln e Netz (2007), Flick (2009), Angrosino (2009), Yin (2010; 2011), Bluhm et al. (2011) e Coraiola et al. (2013).

$\mathrm{Na}$ sequência são descritos os procedimentos metodológicos que foram adotados para a realização desta pesquisa. Por tratar-se de uma bibliometria, procedimento considerado ainda pouco utilizado em pesquisas na área de Administração, no Brasil, bem como por se tratar de um artigo que debate metodologia de pesquisa, procurou-se ser, particularmente, detalhista no relato dos procedimentos metodológicos adotados, de forma a 


\section{WÜNSCH TAKAHASHI}

garantir transparência e clareza para julgamento do leitor com relação aos dados e às análises indicados.

Essa indicação de descrever em detalhes, no relato final da pesquisa, os passos utilizados é referida, por Yin (2005; 2011), como "encadeamento claro de evidências", ou, em seus termos "Clear chain of evidence", também Merriam (1998) aponta a necessidade desse cuidado nos relatos de pesquisa, e cita Lincoln e Guba (1985) e seu conceito de "audit trail", que consiste na necessidade de indicar, nos relatos de pesquisa, as circunstâncias em que os dados foram coletados, como ocorreu a coleta e análise dos dados, incluindo justificações da escolha das técnicas utilizadas, de forma a garantir que o leitor possa identificar, de forma transparente, o passo a passo de realização da pesquisa (GIBBERT; RUIGROK; WIKI, 2008; GIBBERT; RUIGROK, 2010). A ideia, portanto, é permitir que o leitor reconstrua o caminho percorrido pelos pesquisadores, desde as perguntas iniciais de pesquisa até as conclusões que serão apresentadas.

Nas seções seguintes são expostos os resultados e, por conseguinte, as análises destes; e finaliza-se o artigo com as conclusões e considerações finais da pesquisa. 


\section{REFERENCIAL TEÓRICO}

Este trabalho apoiou-se em textos de metodologia qualitativa de pesquisa em Ciências Sociais e Administração, tais como propostos por Triviños (1987), Eisenhardt (1989), Merriam (1998), Creswell (2007), Denzin, Lincoln e Netz (2007), Flick (2009), Angrosino (2009), Yin (2010; 2011), Bluhm et al. (2011) e Coraiola et al. (2013).

Denzin, Lincoln e Netz (2007) descrevem a triangulação como uma ferramenta para assegurar a compreensão em profundidade do fenômeno em estudo, pois compreendem que é impossível captar objetivamente a totalidade dos fenômenos. Desse modo, a triangulação aparece como uma alternativa para a validação de representações da realidade em investigação. Para esses autores, assim como para Flick (2009), em uma mesma investigação, o uso da triangulação de fontes de evidência, métodos, perspectivas e observadores aumenta a qualidade da pesquisa à medida que lhe atribui mais rigor, complexidade e profundidade.

A triangulação na pesquisa qualitativa pode ser vista metaforicamente como a observação de um cristal (DENZIN; LINCOLN; NETZ, 2007), em que se busca compreender um mesmo fenômeno a partir de múltiplas facetas que refletem realidades também múltiplas. Assim, a triangulação constitui um exercício de investigação em torno de um mesmo tema central no qual os dados e as análises de cada faceta procuram criar uma simultaneidade, aproximando a pesquisa da totalidade do fenômeno.

Num esforço semelhante ao feito neste artigo, de sistematizar o entendimento do que é a triangulação e analisar como ela tem sido utilizada na publicação brasileira, Zappellini e Feuerschütte (2015) apresentam a ideia central de como esse conceito é entendido por diferentes autores, conforme segue no Quadro 1: 


\section{WÜNSCH TAKAHASHI}

Quadro 1 Sistematização das concepções de triangulação

\section{$\operatorname{Autor}(\mathrm{ES})$}

Denzin (1970)

Denzin e Lincoln

(2005)

Patton (2002)

Davidson (2005)

Flick (2009a;

2009c; 2013)

Stake $(2005 ; 2011)$

\section{Conceito}

Combinação de metodologias diferentes para anali-

sar o mesmo fenômeno, de modo a consolidar a construção de teorias sociais.

Combinação de diferentes fontes e métodos de coleta de dados.

Combinação de diferentes fontes e métodos de coleta de dados em que a análise desses dados é feita em conjunto, e não considerando dados individuais.

Combinação de diferentes métodos, grupos de estudo, ambientes, período de tempo e perspectivas teóricas para lidar com um fenômeno. Estudo de um tema e um problema de pesquisa com base em duas perspectivas privilegiadas, assumindo diferentes visões a respeito da questão de pesquisa e combinando diferentes tipos de dados sob a mesma abordagem teórica para a produção de mais conhecimento do que seria possível com base em uma só perspectiva.

Método que utiliza dados adicionais para validar ou ampliar as interpretações feitas pelo pesquisador,

nificado por meio da repetição das observações ou interpretações.

Fonte: Zappellini e Feuerschütte (2015, p.246)

Dessa forma, na literatura de metodologia de pesquisa consultada para a realização deste estudo é ponto pacífico, entre os autores, a indicação do uso de dois tipos de triangulação: a triangulação de fontes de evidência e a de métodos de coleta/análise de dados como estratégias metodológicas para aumentar a compreensão sobre um mesmo fenômeno, também, servindo para reforçar a validade da investigação científica. 
A partir da breve contextualização da triangulação como aspecto metodológico que auxilia a realização de pesquisas, apresenta-se os diferentes tipos de tratamentos que lhe são atribuídos.

(i) Triangulação de dados referindo-se à utilização de múltiplas fontes de evidência empírica (como relatos de diferentes informantes, relato do próprio pesquisador que observou diretamente o fenômeno em estudo, documentos, artefatos, etc.).

(ii) Triangulação de métodos ou técnicas, que envolve o uso de diferentes técnicas de coleta das evidências empíricas (entrevistas estruturadas, entrevistas semiestruturadas, grupos focais, questionários, gravações, filmagens, observação direta, observação participante, etc.).

As triangulações de (i) fontes de evidência e (ii) de métodos (técnicas) relacionam-se ao conceito de validade de constructo que procura considerar o quanto um estudo de fato investiga o que diz investigar, isto é, o quão acurado é o procedimento utilizado para observar a realidade (DENZIN; LINCOLN; NETZ, 2007; GIBBERT; RUIGROK; WICKI, 2008; YIN, 2011).

Da leitura desses autores, pressupõe-se, portanto, que triangular fontes de evidência e utilizar diferentes técnicas para acessar e analisar essas fontes permitiria a representação ampliada e aprofundada do objeto ou fenômeno sob investigação.

Além da triangulação de (i) fonte de evidências e (ii) de métodos, elencadas em precedência, existem ainda autores que indicam a realização de (iii) triangulação teórica ou de perspectiva e (iv) a triangulação de investigadores.

(iii) Triangulação teórica diz respeito à utilização de diferentes perspectivas ou abordagens teóricas para analisar os mesmos dados e relaciona-se com a validade interna de uma investigação (GIBBERT; RUIGROK; WICKI, 2008; YIN, 2010). A validade interna é também entendida como validade lógica, ou seja, a capacidade de a pesquisa apresentar argumentos plausíveis e coerentes o suficiente para defender suas conclusões de analíticas (GIBBERT; 
RUIGROK, 2010). Em alguns textos, a triangulação de teorias ou perspectivas corresponde à utilização de diferentes lentes e/ou corpos teóricos tanto na construção do framework da pesquisa como na capacidade de interpretar as evidências encontradas em uma pesquisa com conceitos e teorias (GIBBERT; RUIGROK; WICKI, 2008).

(iv) A triangulação de investigadores, por sua vez, consiste no envolvimento de mais de um pesquisador no processo de pesquisa. Eisenhardt (1989) destaca que a convergência de percepção de mais de um pesquisador atribui confiabilidade aos resultados, análises e conclusões, além de aumentar a capacidade de se chegar a resultados inovadores. Sandberg (2005) concorda com esta ideia ao apontar que a discussão dos dados, resultados e análises por mais de um pesquisador aumenta a validade da pesquisa, indicando que houve triangulação entre pesquisadores toda vez em que um grupo de investigadores, ao analisar os mesmos dados e resultados, chegam às mesmas conclusões.

Por sua vez, a técnica de triangulação proposta por Triviños (1987) diferencia-se das demais, pois propõe uma triangulação por meio de diferentes níveis de análise que compõem um mesmo fenômeno. Para o autor, a técnica de triangulação

[...] tem por objetivo básico abranger a máxima amplitude na descrição, explicação e compreensão do foco em estudo. Parte de princípios que sustentam que é impossível conceber a existência isolada de um fenômeno social, sem raízes históricas, sem significados culturais e sem vinculações estreitas e essenciais com uma macrorrealidade social. (TRIVIÑOS, 1987, p. 38).

Desse modo, o autor propõe que a coleta e análise de dados sobre um determinado tema ou sujeito abordem três níveis distintos: (i) os processos e produtos centrados no sujeito; (ii) os elementos produzidos pelo meio em que o sujeito se encontra inserido; e (iii) os processos e produtos originados 
pela estrutura social, econômica e cultural do macro-organismo social do sujeito, aqui relacionados principalmente ao modo de produção vigente, suas forças e relações de produção (TRIVIÑOS, 1987).

Para a coleta de dados sobre os processos e produtos centrados no sujeito, o autor destaca a utilização de técnicas de coleta de dados primários, tais como entrevistas, observação, questionários, autobiografias e demais técnicas que permitam ouvir a voz do próprio sujeito. Para os elementos produzidos pelo meio, ele indica fontes de dados secundárias, tais como documentos, instrumentos oficiais, estatísticas, imagens e fotografias. Já no tocante aos processos e produtos originados da estrutura social, conquanto o autor não delimite as técnicas de coleta de dados, entende-se que podem ser verificados por meio de todas as técnicas de coleta já citadas e, inclusive, por exemplo, mediante pesquisa histórica, pois se tratam, basicamente, de dados que indicam características do modo de produção vigente e como elas se relacionam com o sujeito e os fenômenos estudados (TRIVIÑOS, 1987). A Figura 1, a seguir, apresenta, de forma esquemática, o entendimento sobre essa técnica de triangulação.

Figura 1 Esquema exemplificativo da Técnica de Triangulação proposta por Triviños (1987)

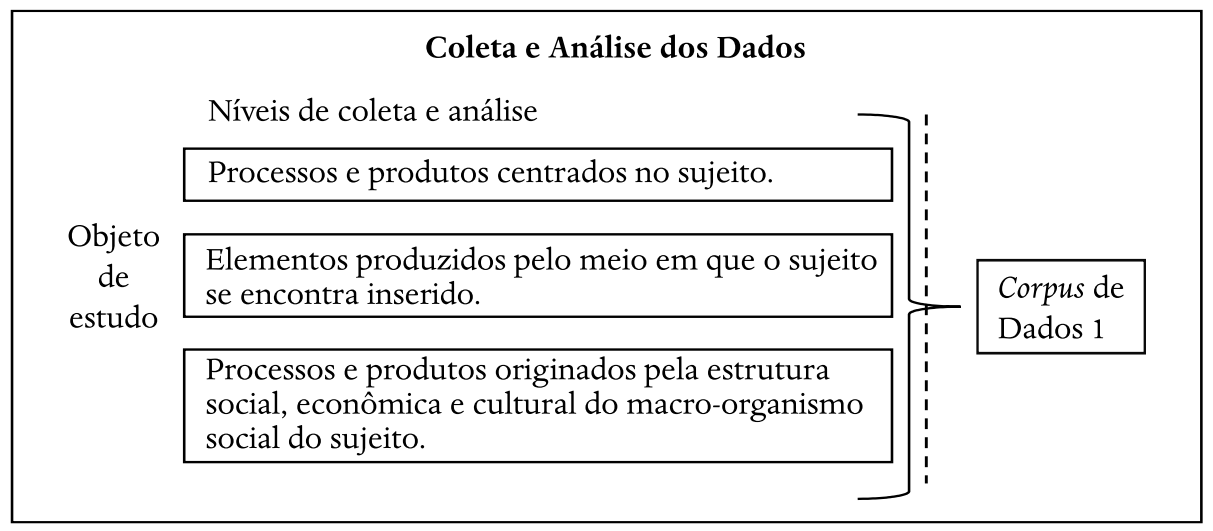

Fonte: Elaborado pelos autores. 
Essa sistematização dos tipos de triangulação e seus autores centrais é convergente com a organizada por Zappellini e Feuerschütte (2015). Esses autores desenvolveram uma análise quanto às publicações brasileiras em Administração considerando triangulação de dados, de investigadores, de teoria e metodológica (dividindo entre "de métodos" e "entre métodos"). Ainda apresentam discussões sobre a existência da triangulação interdisciplinar e analistas múltiplos, mas estas não aparecem na análise dos artigos avaliados no estudo.

É importante sinalizar que a utilização de qualquer uma das formas de triangulação aqui apresentada é passível de aumentar a complexidade de operacionalização da pesquisa, ao mesmo tempo que também contribui para o aumento da sua validade. Ademais, não é objeto de discussão aqui se determinado tipo de triangulação é melhor ou pior que outro, nem se defende que a técnica de triangulação proposta por Triviños (1987) seja superior às demais, ela é tratada, apenas, como uma representante sistematizada de triangulação que tem passos de aplicação específicos. 


\section{PROCEDIMENTO METODOLÓGICO}

Em razão de este artigo refletir sobre metodologia de pesquisa na área de Administração e, como se verá adiante, infere que o relato transparente dos passos utilizados para coleta, tratamento e análise dos dados de uma pesquisa são importantes para sua qualidade e confiabilidade, faz-se especialmente importante mostrar, em detalhes, os procedimentos metodológicos que foram aqui adotados:

Neste trabalho foram analisados artigos que se declaram como estudos de caso publicados em periódicos classificados como A1 até B1 pela CAPES, conforme evento de classificação Qualis 2013, na área de Administração, entre os anos de 2008 e 2012.

A análise foi realizada da seguinte maneira:

(1) Primeiramente foram identificados os periódicos brasileiros que se enquadravam na classificação anteriormente exposta; obteve-se um total de 25 revistas, sendo seus nomes e siglas elencados na Tabela 1.

(2) Realizou-se o download de todos os artigos publicados nesses periódicos no período de 2008 a 2012: totalizando 4.438 artigos (o número de artigos por periódico também pode ser verificado na Tabela 1).

É importante ressaltar que o acesso aos artigos se deu por meio do sistema de indexação de artigos das áreas de Administração, Contabilidade e Turismo, desenvolvido pela Associação Nacional dos Programas de Pós-graduação em Administração (ANPAD), chamado Scientific Periodicals Eletronic Library (SPELL) e, nos casos de revistas não indexadas nesse sistema, pelo Scientific Electronic Library Online (Scielo) - outro sistema indexador de artigos científicos.

Além disso, foram inclusas na análise somente as revistas brasileiras que disponibilizam seu conteúdo on-line e gratuitamente, bem como que tratam de temas correlatos à administração e gestão, isto é, foram excluídas da análise aquelas que têm como escopo Contabilidade, Economia e Psico- 
logia, ainda que classificadas pela Capes como periódicos que pontuam na área de Administração.

Cabe ressaltar ainda que a Revista Brasileira de Gestão de Negócios (classificada como B1, conforme Qualis 2013) apresentou problemas no momento da coleta dos artigos, e, portanto, não foi inclusa na análise.

A opção de coletar todos os artigos publicados nesses cinco anos e "baixá-los" integralmente no formato PDF, em detrimento de apenas escolher os artigos que mencionassem os termos "estudo de caso" e "triangulação” em seus títulos, ou sessões de resumo e/ou palavras-chave - o que poderia ser realizado diretamente nas opões de busca disponibilizados pelos sistemas de indexação consultados - deu-se por considerar que essas informações podem estar alocadas em todo o corpo do texto, e não somente nessas seções citadas.

(3) Uma vez formada a base de dados de 4.438 artigos em formato de arquivo PDF, realizou-se a importação destes para o software de tratamento de pesquisa qualitativa denominado NVivo (na versão 9) e lá os artigos foram organizados respectivamente nas pastas correspondentes à sua revista.

(4) Por meio da ferramenta consulta de texto, foram identificados todos os artigos que continham o termo "estudo de caso" ou as seguintes variações: "casos múltiplos", "comparativo de casos" e "multicasos". Foram identificados 1.384 artigos, conforme se pode visualizar na Tabela 1.

Cabe ressaltar que um cuidado metodológico adotado foi a realização da leitura das sessões de metodologia desses 1.384 artigos com o objetivo de identificar se a incidência do termo "Estudo de Caso" e seus correlatos, como citado anteriormente, identificados pelo software de fato se referiam à estratégia de pesquisa adotada pelo artigo ou se tratava de utilização do termo em outro contexto (como no título de alguma referência, por exemplo).

(5) Na sequência foram identificados, dentre os artigos que relatavam ser estudos de caso, a incidência do termo "triangulação", bem como suas possíveis variações, como "triangulações", 
"triangular", "triangulei", "triangulamos", "triangulou-se" e "foram triangulados". Disso resultaram 167 artigos que se considera que caracterizam estudos de caso que apresentam algum tipo de informação sobre triangulação.

Tabela 1 Quantitativo de artigos, estudos de caso e relatos de triangulação por revista analisada

\begin{tabular}{|c|c|c|c|c|}
\hline Nome da Revista & $\begin{array}{c}\text { SIGLA } \\
\text { ADOTADA }\end{array}$ & Artigos & $\begin{array}{l}\text { Estudo } \\
\text { DE CAso }\end{array}$ & $\begin{array}{c}\text { ESTUDOS DE } \\
\text { CASO COM } \\
\text { RELATO DE }\end{array}$ \\
\hline & & & & TRIANGULAÇ̃̃O \\
\hline Ambiente e Sociedade & $A \& S$ & 135 & 25 & 1 \\
\hline Brazilian Business Review & BBR & 109 & 18 & 0 \\
\hline $\begin{array}{l}\text { Contabilidade, Gestão e } \\
\text { Governança }\end{array}$ & CGG & 113 & 43 & 1 \\
\hline Caderno CRH & $\mathrm{CRH}$ & 241 & 21 & 1 \\
\hline Cadernos Ebape.br & EBAPE & 274 & 70 & 13 \\
\hline Gestão e Produção & GESTPROD & 293 & 159 & 14 \\
\hline Gestão e Planejamento & GESTPLAN & 103 & 48 & 6 \\
\hline $\begin{array}{l}\text { Organizações \& } \\
\text { Sociedade }\end{array}$ & O\&S & 221 & 72 & 10 \\
\hline Pesquisa Operacional & $\mathrm{PO}$ & 53 & 15 & 3 \\
\hline Produção & PRODU & 242 & 137 & 14 \\
\hline $\begin{array}{l}\text { Revista de Administração } \\
\text { Contemporânea }\end{array}$ & RAC & 266 & 83 & 17 \\
\hline $\begin{array}{l}\text { Revista de Administração } \\
\text { Mackenzie }\end{array}$ & RADMACK & 273 & 72 & 7 \\
\hline $\begin{array}{l}\text { Revista de Administração } \\
\text { de Empresas }\end{array}$ & RAE & 172 & 56 & 14 \\
\hline $\begin{array}{l}\text { Revista de Administração } \\
\text { e Inovação }\end{array}$ & RAI & 158 & 73 & 5 \\
\hline $\begin{array}{l}\text { Revista de Administração } \\
\text { (FEA-USP) }\end{array}$ & RAUSP & 172 & 55 & 9 \\
\hline
\end{tabular}




\begin{tabular}{|c|c|c|c|c|}
\hline $\begin{array}{l}\text { Revista Portuguesa e } \\
\text { Brasileira de Gestão }\end{array}$ & RPBG & 111 & 23 & 7 \\
\hline $\begin{array}{l}\text { Revista de Ciências da } \\
\text { Administração }\end{array}$ & RCIADM & 156 & 75 & 9 \\
\hline $\begin{array}{l}\text { Dados Revista de Ciências } \\
\text { Sociais }\end{array}$ & RDADOS & 141 & 10 & 0 \\
\hline $\begin{array}{l}\text { Revista de Administração } \\
\text { da Universidade Federal } \\
\text { de Santa Maria }\end{array}$ & RAUF & 172 & 61 & 6 \\
\hline $\begin{array}{l}\text { Revista Eletrônica de } \\
\text { Administração }\end{array}$ & READ & 151 & 59 & 7 \\
\hline $\begin{array}{l}\text { Revista Brasileira de } \\
\text { Finanças }\end{array}$ & $\mathrm{RBF}$ & 100 & 5 & 0 \\
\hline $\begin{array}{l}\text { Revista de Administração } \\
\text { Pública }\end{array}$ & RAP & 306 & 100 & 16 \\
\hline $\begin{array}{l}\text { Revista de Contabilidade } \\
\text { e Organizações }\end{array}$ & $\mathrm{RCO}$ & 122 & 43 & 3 \\
\hline Novos Estudos CEBRAP & CEBRAP & 187 & 5 & 0 \\
\hline \multirow[t]{2}{*}{$\begin{array}{l}\text { Revista de Administração } \\
\text { da FACES }\end{array}$} & FACES & 167 & 56 & 4 \\
\hline & TOTAIS & 4438 & 1384 & 167 \\
\hline
\end{tabular}

Fonte: Elaborado pelos autores.

(6) Finalmente, todos os 167 artigos identificados foram lidos por três pesquisadores. E essa triangulação de pesquisadores foi realizada da seguinte maneira: individualmente, cada um leu e preencheu um check-list sobre cada artigo, então, assinalando a presença dos cinco tipos de triangulação aqui trabalhados, quais sejam: triangulação de dados/fontes, triangulação de métodos, triangulação de pesquisador, triangulação de teoria e/ou a técnica de triangulação - conforme proposta por Trivinõs -, bem como se a citação de tal triangulação era autoevidenciada (declarada explicitamente no texto) ou se podia ser inferida pela leitu- 
ra. Na sequência, foram comparadas as classificações realizadas pelos três pesquisadores; nos casos em que há divergências, a sessão de metodologia do respectivo artigo foi relida, e, por meio de debate e consenso entre os três pesquisadores, reclassificado o material.

Faz-se necessário justificar a necessidade de identificar se as triangulações analisadas eram autoevidenciadas nos textos ou inferidas da leitura: assim, partindo de análise feita em um estudo piloto (em que foram analisados 20 artigos randomicamente selecionados), percebeu-se existirem duas possibilidades de relato para cada modalidade de triangulação:

(a) aquela em que os autores do artigo declaram a utilização de alguma(s) forma(s) específica(s) de triangulação, declarando-a explicitamente em alguma parte de seu artigo; e

(b) a possibilidade de os autores relatarem seus procedimentos metodológicos indicando a utilização de triangulação, sem, no entanto, usar especificamente esse termo. São exemplos de passagens em que se pode inferir que, na pesquisa, utilizou-se triangulação: "utilizaram-se múltiplas fontes de dados", "utilizaram-se entrevista e grupo focal", "os dados foram analisados separadamente por três pesquisadores", "duas teorias de natureza crítica embasam as análises", entre outras construções textuais.

Assim, na análise dos estudos de caso, identificou-se o uso de triangulação tanto quando declarada pelos autores dos artigos (o que se considerou como "autoevidenciada", conforme Tabela 2) quanto quando possível de ser inferida pela leitura do trabalho (o que se considerou como "inferida pela leitura", termo também utilizado na Tabela 2 ).

Segue um exemplo da análise realizada: tome-se a seguinte passagem de um dos artigos:

[...] utilização de triangulação metodológica à base de uso de múltiplas fontes de informação (gerentes, engenheiros, técnicos, material publicado das empresas) e de diversas técnicas de coleta de informação (entrevistas, 
encontros fortuitos, observação direta). (ARTIGO ANALISADO 1, grifo nosso).

A análise da passagem supratranscrita permite identificar o que é aqui entendido como uma declaração de uso de triangulação metodológica e também o que se está considerando como uso de triangulação (neste caso, de fontes de evidência), que pode ser inferido pela leitura do texto, ainda que não declarada explicitamente por seus autores. Essas informações, como se verá nas conclusões deste trabalho, são relevantes para tecer considerações sobre o tipo de relato que se faz do uso de triangulação em estudos de caso na área de Administração no Brasil.

Aqui se aponta uma limitação do procedimento metodológico adotado nesta pesquisa: se nos artigos selecionados foi possível verificar que, em alguns casos, o uso de triangulação pode ser verificado por inferências, e não declarada explicitamente nos artigos, ou seja, sem os autores terem utilizado a palavra triangulação, é de se esperar que entre os demais artigos publicados entre 2008 e 2012 que não foram selecionados para a análise por não possuírem a palavra triangulação, isso também tenha acontecido. A transposição dessa limitação só será possível com a leitura na íntegra de todos os artigos publicados nesse período, caracterizando, portanto, o estudo que aqui se apresenta como um recorte preliminar que consegue apenas analisar artigos que explicitamente declaram ter realizado alguma forma de triangulação na área de Administração, nos 5 anos em análise, não abrangendo sua totalidade. Defende-se, todavia, que, ainda com essa limitação, um estudo desta natureza é relevante para a reflexão sobre a prática de pesquisa em Administração no Brasil. 


\section{RESULTADOS E ANÁLISES}

A análise bibliométrica realizada permitiu identificar o número de artigos por periódico que relatava se tratar de estudos de caso (resultados apresentados na Tabela 1), bem como o número de artigos que utilizou algum ou mais de um dos cinco tipos de triangulação anteriormente citados; esses resultados podem ser visualizados na Tabela 2 .

Tabela 2 Quantitativo de artigos que utilizaram cada um dos cinco tipos de triangulação por revista analisada

\begin{tabular}{|c|c|c|c|c|c|c|c|c|c|c|c|c|}
\hline \multirow[b]{2}{*}{ Total } & & \multirow{2}{*}{ 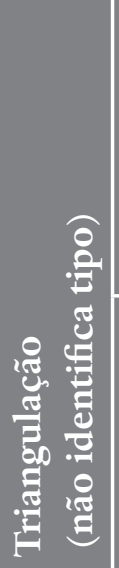 } & \multicolumn{2}{|c|}{ 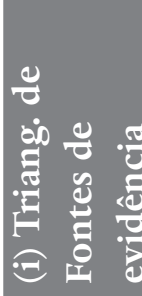 } & \multicolumn{2}{|c|}{ 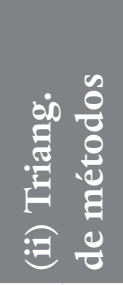 } & \multicolumn{2}{|c|}{ 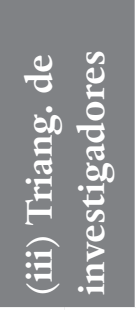 } & \multicolumn{2}{|c|}{ 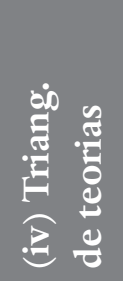 } & \multicolumn{2}{|c|}{ 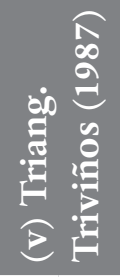 } \\
\hline & & & $\begin{array}{l}\frac{5}{8} \\
\frac{8}{0} \\
\frac{0}{5} \\
\frac{8}{8} \\
\frac{0}{3}\end{array}$ & 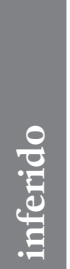 & 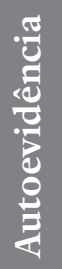 & 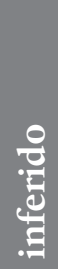 & $\begin{array}{l}\frac{5}{8} \\
\frac{8}{0} \\
\frac{0}{5} \\
\frac{8}{8} \\
\frac{8}{3}\end{array}$ & 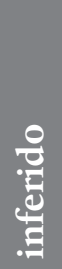 & 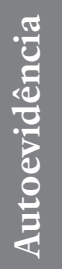 & 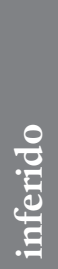 & 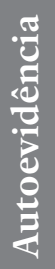 & $\begin{array}{l}\stackrel{0}{0} \\
\text { : } \\
.\end{array}$ \\
\hline GESTPROD & 9 & 4 & 0 & 9 & 3 & 7 & 0 & 0 & 0 & 0 & 1 & 0 \\
\hline GESTPLAN & 5 & 1 & 3 & 2 & 0 & 5 & 0 & 0 & 0 & 0 & 0 & \\
\hline RADMACK & 5 & 2 & 2 & 2 & 0 & 2 & 0 & 0 & 0 & 0 & 0 & \\
\hline RBPG & 4 & 1 & 2 & 1 & 2 & 1 & 0 & 1 & 1 & 0 & 0 & 0 \\
\hline RCIADM & 4 & 0 & 2 & 4 & 1 & 5 & 0 & 0 & 0 & 0 & 0 & 0 \\
\hline PRODU & 10 & 0 & 10 & 0 & 1 & 1 & 2 & 0 & 0 & 0 & 0 & 0 \\
\hline EBAPE & 11 & 0 & 7 & 3 & 1 & 8 & 0 & 0 & 1 & 0 & 0 & 0 \\
\hline RAC & 12 & 3 & 7 & 5 & 2 & 9 & 2 & 0 & 1 & 1 & 1 & 0 \\
\hline$A \& S$ & 1 & 0 & 1 & 0 & 1 & 0 & 0 & 0 & 0 & 0 & 0 & 0 \\
\hline CGG & 0 & 0 & 0 & 0 & 0 & 0 & 0 & 0 & 0 & 0 & 0 & 0 \\
\hline $\mathrm{CRH}$ & 0 & 0 & 0 & 1 & 0 & 1 & 0 & 0 & 0 & 0 & 0 & 0 \\
\hline
\end{tabular}




\begin{tabular}{lcccccccccccc} 
PO & 0 & 0 & 0 & 0 & 0 & 0 & 0 & 0 & 0 & 0 & 0 & 0 \\
RAE & 10 & 0 & 9 & 1 & 2 & 8 & 1 & 1 & 0 & 0 & 0 & 1 \\
RAI & 5 & 1 & 4 & 1 & 1 & 4 & 0 & 0 & 0 & 0 & 0 & 0 \\
RAUSP & 4 & 1 & 2 & 3 & 0 & 3 & 0 & 1 & 0 & 0 & 1 & 0 \\
O\&S & 9 & 4 & 3 & 6 & 4 & 3 & 0 & 0 & 2 & 0 & 0 & 0 \\
FACES & 1 & 0 & 0 & 1 & 1 & 0 & 0 & 0 & 0 & 0 & 0 & 0 \\
RAUF & 5 & 1 & 4 & 1 & 4 & 1 & 0 & 0 & 0 & 0 & 0 & 0 \\
READ & 5 & 0 & 5 & 0 & 2 & 3 & 0 & 0 & 0 & 0 & 0 & 0 \\
RAP & 12 & 3 & 4 & 7 & 5 & 6 & 0 & 1 & 0 & 1 & 0 & 0 \\
RCO & 1 & 0 & 1 & 0 & 0 & 1 & 0 & 0 & 0 & 0 & 0 & 0 \\
TOTAIS & $113^{\star}$ & $\mathbf{2 1}$ & $\mathbf{6 6}$ & $\mathbf{4 7}$ & $\mathbf{3 0}$ & $\mathbf{6 8}$ & $\mathbf{5}$ & $\mathbf{4}$ & $\mathbf{5}$ & $\mathbf{2}$ & $\mathbf{3}$ & $\mathbf{1}$ \\
\hline
\end{tabular}

Total de citações de "triangulação" autoevidenciadas pelos autores em seus artigos.

Total de relatos de triangulação possíveis de serem inferidos pela leitura dos artigos.

Total acumulado de utilização de triangulação (autoevidenciado + inferido)

* Dos 167 artigos inicialmente selecionados por conterem tanto o termo "Estudo de Caso", como o termo "triangulação", conforme apresentado na Tabela 1, foram excluídos 54 artigos que utilizavam o termo fora de contexto ou com outro sentido que não uma estratégia metodológica, o que só pôde ser identificado após a leitura dos trabalhos na íntegra, restando, portanto, um total de 113 artigos analisados.

Fonte: Elaborado pelos autores.

A primeira análise que pode ser realizada diz respeito aos 113 artigos de Estudo de Caso que relatam triangulação, esse número representa aproximadamente $8,16 \%$ do total dos artigos publicados que declaram utilizar essa estratégia de pesquisa no período de cinco anos analisado, permitindo inferir que apenas uma minoria dos estudos de caso publicados na área de Administração nesse período relata a utilização de triangulação.

Este resultado é contraintuitivo, visto que o estudo de caso é o delineamento de pesquisa mais utilizado na academia de Administração 
brasileira, conforme Coraiola et al. (2013), e que a triangulação, como visto na sessão de referencial teórico deste trabalho, é um critério de rigor metodológico bastante difundido nos textos de metodologia de pesquisa da área.

Os resultados, apresentados na Tabela 2, permitem verificar que nos 113 artigos analisados e que indicavam de alguma forma terem realizado triangulação como uma ação concreta de pesquisa, que o tipo de triangulação mais citado foi o de (i) fontes de evidências (citado em 66 dos 113 artigos); em segundo lugar, a (ii) triangulação de métodos (citada explicitamente em 30 dos 113 artigos); e com menor incidência, em terceiro lugar, empatadas a (iii) triangulação de investigadores (citada em cinco dos 113 artigos) e (iv) triangulação de teoria (também citada em cinco dos 113 artigos).

Se forem verificados os artigos que indicaram ter utilizado triangulação, porém sem explicitar a técnica de triangulação, tem-se: a (i) triangulação de fontes de evidência sendo utilizada em 113 artigos, a (ii) triangulação de métodos em 98 artigos, a (iii) triangulação de pesquisadores em nove, a (iv) triangulação de teoria ou perspectiva em sete e a (v) técnica de triangulação de Triviños (1987) em quatro artigos.

Importante apontar que em 21 artigos ainda foi possível identificar a utilização do termo triangulação de uma maneira genérica, ou seja, como uma estratégia ou ação concreta de pesquisa, sem que os autores apresentem quaisquer indicativos que permitam ao leitor indicar a forma específica de triangulação realizada.

Ao considerar a utilização concreta de triangulação de fontes de evidência, isto é, considerando-se os artigos cujos autores mostraram preocupação em relatar a utilização concreta de triangulação rotulando ou não essa ação como triangulação, tem-se para (i) triangulação de fontes de evidência, 66 artigos com autoevidenciação e 47 com indicações de realização de triangulação que podem ser inferidas pela leitura, totalizando 113 artigos. Esse número representa que $100 \%$ dos artigos analisados que relatam ou mostram terem realizado triangulação, utilizaram, ao menos, (i) triangulação de fontes de evidência. 
Já em relação à (ii) triangulação de métodos, observou-se que esta modalidade foi menos autoevidenciada por parte dos autores, sendo que 30 artigos utilizam o rótulo "triangulação de métodos", "triangulação de técnicas" ou equivalente. No entanto 68 artigos descrevem a utilização concreta de múltiplos métodos de coleta e análise de dados não usando, porém, o termo explicitamente. Disso tem-se que 98 artigos, isto é, $86,72 \%$ dos artigos analisados mostram terem realizado triangulação metodológica, ainda que não tenham adotado o termo.

Pôde-se verificar, portanto, que os estudos de caso publicados recentemente por periódicos considerados de qualidade na área de Administração, no Brasil, e que relatam a realização de triangulação revelam em sua totalidade preocupação em relatar ou mostram a utilização de ações concretas de pesquisa relacionadas à (i) triangulação de fontes de evidência para afirmar a validade dos dados e resultados por eles apresentados. A maioria deles $(86,72 \%)$ apresenta também preocupação em assinalar a utilização de (ii) triangulação de métodos de coleta/análise de dados. Verificou-se assim que a preocupação recai essencialmente na questão de assegurar validade de constructo aos estudos de caso, que diz respeito a quanto um estudo de fato investiga o que diz investigar, isto é, o quão acurado é o procedimento utilizado para observar a realidade (DENZIN; LINCOLN; NETZ, 2007; GIBBERT; RUIGROK; WICKI, 2008; YIN, 2011).

Entre os autores que relatam explicitamente a utilização de triangulação de pesquisadores e de teoria não é atribuída mesma ênfase. Verificou-se que mesmo considerando os casos em que essas ações concretas de pesquisa podem ser inferidas da metodologia descrita, ainda que não autoevidenciada pelos autores, apenas nove dos 113 artigos mostraram preocupação em relatar a utilização de (iii) múltiplos investigadores, e apenas cinco de explicitar a utilização de (iv) múltiplas perspectivas ou teorias. Desse modo, pode-se inferir que esses tipos de triangulação não representam ações concretas de pesquisa muito utilizadas em estudos de caso na área de Administração, no Brasil. Foi possível perceber, portanto, que a preocupação com a validade interna e com a confiabilidade não representam aspectos relevantes nos relatos de procedimentos metodológicos dos artigos analisados 
(DENZIN; LINCOLN; NETZ, 2007; GIBBERT; RUIGROK; WICKI, 2008; YIN, 2011).

Interessante apontar também que, dos 113 artigos analisados, apenas sete relatam explicitamente a utilização de mais de um tipo de triangulação combinada, sendo que apenas um declara ter utilizado as quatro modalidades básicas (triangulação de fontes, métodos, investigador e teoria):

Patton (1987 como citado em Yin, 1994, p. 92) aponta quatro tipos de triangulação, que é um método que envolve o uso de múltiplas fontes de informações: (a) de fontes de dados; (b) entre diferentes avaliadores; (c) de perspectivas a respeito do mesmo fenômeno, também conhecida como triangulação teórica; e (d) de métodos. Para este trabalho, buscou-se abranger os quatro tipos de triangulação, sendo o terceiro utilizado principalmente para a elaboração das proposições já apresentadas. (ARTIGO ANALISADO 3).

Vale ressaltar que não necessariamente porque a triangulação foi explicitamente relatada ela tenha de fato sido utilizada tal como pretendiam os autores que a indicaram. Pois não existe nenhum indício concreto de como foi o uso das técnicas. Essa análise pode ser inferida também do volume de artigos que utilizam o termo "triangulação" de uma maneira genérica (um total de 21 artigos), não indicando que tipo de triangulação foi realizada.

Em alguns casos que se menciona triangulação de forma genérica foi possível inferir como ela foi realizada pela leitura do texto completo, e não pela indicação clara dos autores do artigo. Assim, não se sabe se não há uma clareza conceitual das formas possíveis de realizar uma triangulação ou se os autores consideram esses aspectos tão resolvidos no meio acadêmico a ponto de não haver necessidade de se ater aos pormenores e optam por apenas informar que houve algum tipo de triangulação. Como exemplo, o artigo analisado 7 informa ter usado entrevistas, observação e análise documental - ou seja: parece haver triangulação de fonte de dados e de método. No artigo analisado 8, os autores informam que, para aumentar a validade interna, fizeram triangulação procurando outras fontes de evidência além 


\section{WÜNSCH TAKAHASHI}

de entrevistas (dados secundários) e, já na sequência, informam que a triangulação ocorreu nas entrevistas por terem falado com fornecedores e compradores. Assim, nesse caso também é possível inferir que há triangulação de fontes de dados e de métodos.

Levanta-se, então, a possibilidade de estar ocorrendo disparidade por parte dos pesquisadores quanto ao que consideram triangulação e o que os teóricos de metodologia consideram como triangulação. Como exemplo dessa inadequação no tocante ao que consiste um relato claro da utilização da ação concreta de pesquisa denominada de triangulação pode ser citado:

Os objetivos do trabalho foram analisados por uma óptica descritiva, cujos procedimentos técnicos envolveram levantamento bibliográfico, realização de entrevistas, análise documental, análise de conteúdo e triangulação das informações teóricas com os dados empíricos (FLICK, 2009). Tais considerações permitem afirmar ainda que a pesquisa apresentada foi desenvolvida por uma estratégia de Estudo de Caso (YIN, 2005). (ARTIGO ANALISADO 4).

No exemplo supratranscrito, a forma como os autores utilizam o termo triangulação não deixa claro como de fato essa ação foi efetivada na pesquisa, tampouco com que finalidade. Exemplos como esse são comuns nos casos de artigos que relatam triangulação de uma maneira genérica (21 artigos, do total de 112 , isto é, em 11,61\% dos casos).

Outro exemplo vem de um artigo que cita um trecho de Triviños, conceituando triangulação e, depois, alegando que esse procedimento aumenta a confiabilidade. No entanto os procedimentos relatados não indicam que houve a realização de uma triangulação conforme o que propõe esse autor. O trecho a seguir mostra que os autores do artigo analisado indicam terem realizado uma triangulação de fontes e de métodos, o que não converge com a proposta de Triviños:

Esta permite alcançar “(...) a máxima amplitude na descrição, explicação e compreensão do objeto da pesquisa”. (TRIVINÕS, 1987:138). Jick (1979) aponta que a triangulação aumenta a confiabilidade dos dados e potencializa o que os antropólogos chamam de "trabalho holístico". As- 
sim, em vários momentos foi possível o exercício da triangulação de dados, mormente, ao se comparar os dados provenientes das entrevistas e documentos analisados, bem como, a triangulação de dados entre as próprias entrevistas. (ARTIGO ANALISADO 5)

Assim, parece haver apropriações de trechos conceituais mais genéricos de autores que versam sobre triangulação sem necessariamente haver aprofundamento nos pormenores indicados e defendidos por estes autores. Nesse caso, especificamente, a indicação bibliográfica da citação nem converge com o que consta nas referências ao final do texto (cita-se nessa seção final Triviños, 1992 - e não Triviños, 1987).

Por fim, com relação especificamente à técnica de triangulação indicada por Triviños (1987), verificou-se que foi declaradamente utilizada em apenas quatro estudos de caso. No entanto, perceberam-se inadequações quanto à sua utilização, como no seguinte exemplo:

Para análise dos dados obtidos, utilizou-se a técnica de triangulação sugerida por Triviños (1987), que, em linhas gerais, consiste no emprego de diferentes evidências para busca de uma resposta mais aprofundada (ARTIGO ANALISADO 6).

Como apresentada na revisão de literatura deste trabalho, a técnica de triangulação proposta por Triviños (1987) refere-se à análise de um mesmo fenômeno por meio de dados coletados a partir de fontes de evidências situadas em níveis distintos, o nível do sujeito, o nível do contexto imediato em que o sujeito está inserido, e o nível do contexto mais macro, do sistema de produção vigente (no caso da citação apresentada verifica-se o relato de utilização incompleta da técnica). 


\section{CONCLUSÕES E CONSIDERAÇÕES FINAIS}

Este artigo realizou uma análise do uso das técnicas de triangulação, entendidas como um critério para atribuir rigor metodológico para pesquisas qualitativas, nos estudos de caso publicados pelos periódicos científicos brasileiros, na área de Administração, no período compreendido entre 2008 e 2012. O tempo necessário para processamento e análise das informações, principalmente, para submissão, aceite e publicação do artigo em um meio público de divulgação acadêmica, traz como limitação o fato de o trabalho chegar ao leitor com certa defasagem. Assim, destaca-se a necessidade de realização de pesquisa futura no sentido de compreender os cuidados que as pesquisas na área de Administração têm tomado para relatar e garantir o rigor metodológico em seus trabalhos.

Considerada essa limitação, defende-se ainda assim que este trabalho traz importantes conclusões que permitem refletir sobre como a área de Administração tem utilizado e relatado a triangulação como estratégia metodológica, além de destacar cuidados que podem auferir maior rigor e confiabilidade às pesquisas da área.

No que se refere ao uso das técnicas de triangulação, foi possível perceber que o relato primário (autoevidenciação) sobre a sua aplicação nas pesquisas é pouco indicado, pois apenas $12 \%$ dos artigos que relatam ser estudos de caso utilizam a palavra triangulação para mencionar o utilizo dessa técnica, e, entre esses, verificou-se que essa prática não representa a inserção de informações relevantes sobre como as técnicas foram de fato aplicadas.

Nos estudos de caso em que foi possível identificar como a triangulação foi realizada, verificou-se que a totalidade (100\%) utilizou a triangulação de fontes e grande parte $(86,72 \%)$ utilizou a triangulação de métodos; apenas uma minoria utilizou triangulação de investigadores (4,42\%, isto é cinco artigos), de teorias (também 4,42\%, isto é cinco artigos) e a técnica de triangulação proposta por Triviños (1987), utilizada em apenas quatro estudos de caso $(3,53 \%$ do total).

Os resultados permitiram verificar que há artigos que se preocupam em citar o uso de triangulação, mas não mostram em qual aspecto, como ou onde elas foram aplicadas (em 19,46\% do total isso ocorre). 
Uma vez que esta pesquisa verificou diversas inadequações no relato da triangulação, cabe, como contribuição teórica e prática deste trabalho, elencar quais os equívocos mais comuns e apresentar sugestões de como superá-los:

Apesar do uso combinado dos dois primeiros tipos de triangulação, quais sejam (i) triangulação de fontes de evidência - em 100\% dos trabalhos analisados, e de (ii) triangulação de métodos - em $86,72 \%$, ser recorrentemente indicado nos trabalhos analisados, é possível conceber pesquisas que usam cada tipo de triangulação separadamente, sem, necessariamente realizar os demais.

Imagine-se, como exemplo, uma pesquisa que triangule somente fontes de evidência e que utilize apenas uma técnica de coleta de dados; esta possibilidade é exemplificada na Figura 2, a seguir:

Figura 2 Exemplo de triangulação exclusivamente de fontes de evidência

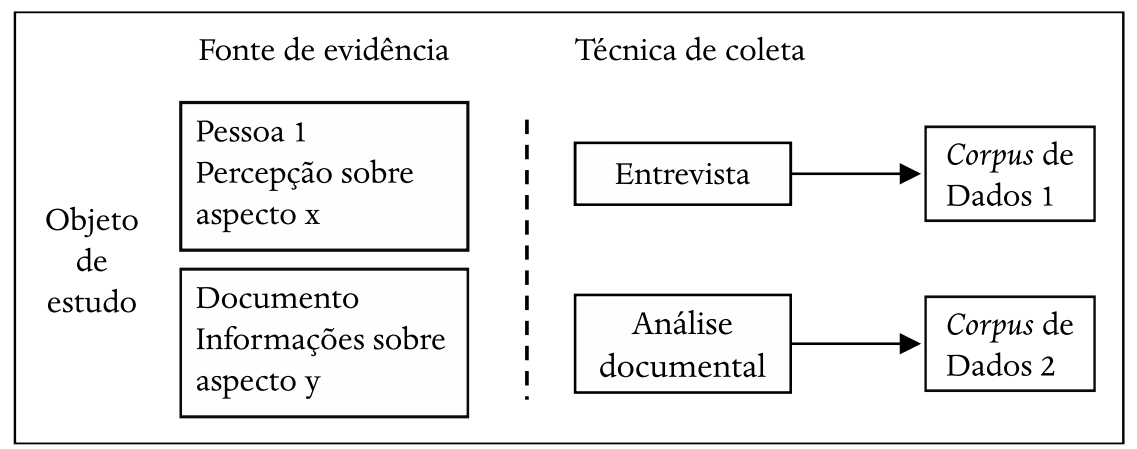

Fonte: Elaborado pelos autores.

Na Figura 2, exemplifica-se o uso da entrevista como técnica única de coleta de dados para diversas pessoas (fontes de evidência) a fim de levantar um mesmo corpus de dados.

Coraiola et al. (2013) descrevem que a lógica de triangulação de fontes e de métodos parte do pressuposto de dados coletados em diferentes fontes por meio de múltiplas técnicas, quando comparados entre si, permitiriam verificar a existência de correspondência entre eles e, à medida 


\section{WÜNSCH TAKAHASHI}

que forem convergentes, podem ser considerados válidos; nesse sentido, sugere-se que a realização combinada de triangulação tanto de fontes de evidência quanto de métodos de coleta e análise de dados atribuiria maior rigor metodológico a uma investigação.

Faz-se necessário, no entanto, apontar um equívoco comum: quando uma pesquisa relata ter utilizado diferentes métodos de coleta de dados, essa informação, por si só, não é suficiente para que o leitor julgue se foi ou não realizada triangulação, tampouco o tipo de triangulação especificamente feita.

Tenha-se, por exemplo, um estudo de caso que declare, em seus procedimentos metodológicos, ter utilizado entrevista e análise documental. Essa informação, em si mesma, não permite inferir se realizou triangulação de fontes de evidência e de métodos.

Se a análise documental foi utilizada para construir uma contextualização do caso e as entrevistas para coletar informações sobre o fenômeno estudado em si, não se pode afirmar que houve triangulação de fontes, pois cada fonte de evidência foi utilizada para coletar dados sobre aspectos diferentes da realidade, e não sobre o mesmo aspecto. Esse exemplo é esquematizado na Figura 3:

Figura 3 Exemplo de utilização de múltiplas fontes e múltiplos métodos sem triangulação

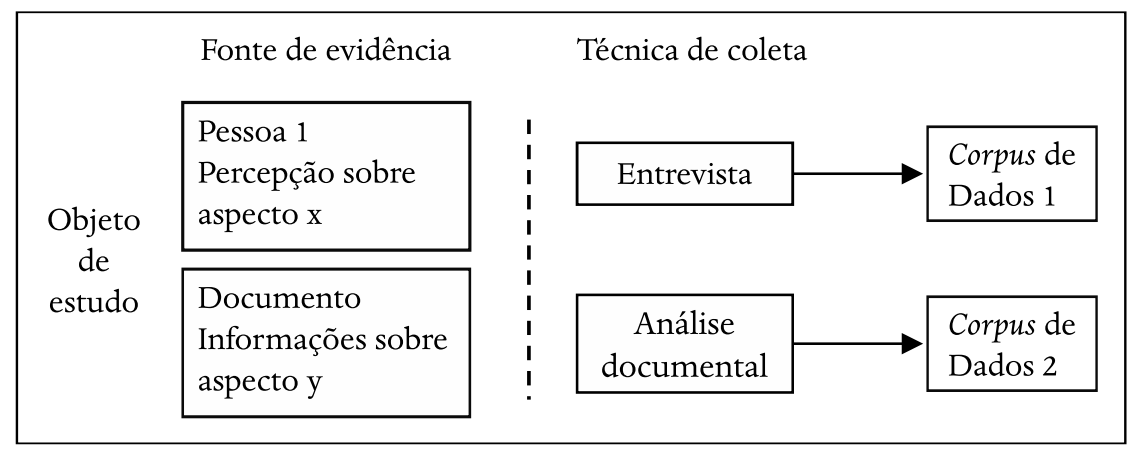

Fonte: Elaborado pelos autores. 
Só se pode assumir a triangulação de fontes de evidência se (a) tanto por meio da entrevista quanto da análise documental a coleta de dados ocorreu sobre um mesmo aspecto e, além disso, (b) se os dados provenientes de diferentes fontes forem comparados para buscar convergências e divergências entre eles. A Figura 4 sintetiza um exemplo desse entendimento:

Figura 4 Exemplo de utilização de triangulação de fontes de evidência com base em entrevistas e análise documental

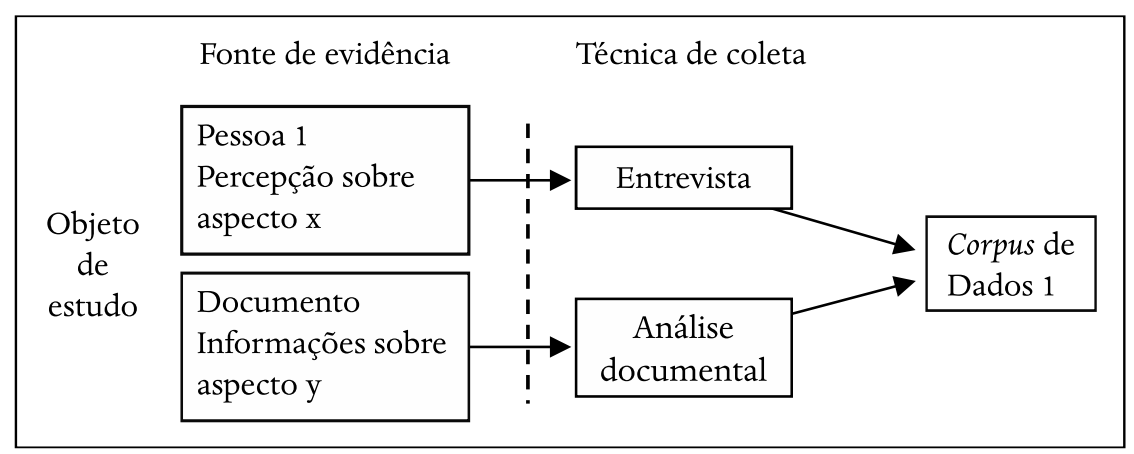

Fonte: Elaborado pelos autores.

É importante sinalizar que, no exemplo apresentado na Figura 4, a triangulação dos dados utiliza múltiplas fontes de evidência, cada uma coletada por meio de um só instrumento, havendo triangulação de fontes, mas não de método (apesar de as técnicas de coleta serem diferentes entre si, elas não analisam a mesma fonte).

Neste ponto, a reflexão analítica começa a mostrar a complexidade do conceito de triangulação: somente o uso de múltiplos métodos de coleta para acessar dados provenientes de uma mesma fonte caracteriza a triangulação de métodos, por exemplo, se, para coletar os dados que os entrevistados podiam fornecer, forem utilizados de maneira combinada mais de um método, tais como questionário, grupo focal, entre outros. A Figura 5 , a seguir, ilustra esse exemplo, que é diferente daquele apresentado na Figura 4. 


\section{WÜNSCH TAKAHASHI}

Figura 5 Exemplo de utilização de triangulação de fontes e de métodos

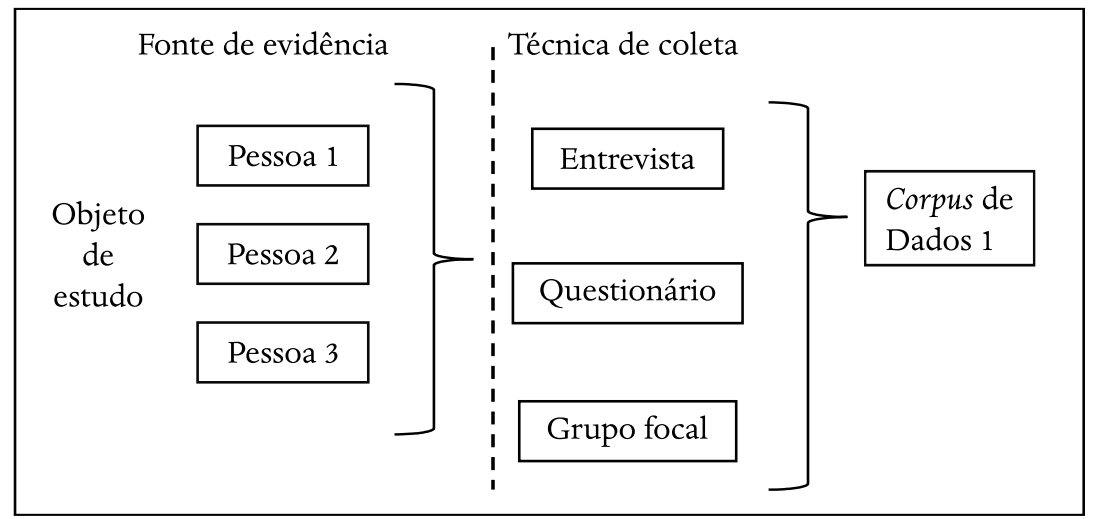

Fonte: Elaborado pelos autores

Não se pode considerar, entretanto, que a afirmação de utilização de múltiplos métodos de coleta de dados, como entrevista e análise documental, configure a aplicação da técnica de triangulação de métodos, uma vez que cada método poderá estar orientado para analisar uma fonte de evidência distinta, nesse caso, não configurando a triangulação (caso relatado na Figura 3). Desse modo, embora, em textos de metodologia, a triangulação de métodos possa ser apresentada em conjunto com a de fontes de evidência, não se pode deixar de considerar que a triangulação de métodos de fato só pode ocorrer dentro do escopo de um só conjunto de fontes de evidência.

Este trabalho permitiu, pois, verificar que a triangulação, embora amplamente indicada pela teoria de metodologia qualitativa, é pouco utilizada em pesquisas do tipo estudo de caso na área de Administração nacional. Nos poucos casos em que foi utilizada, os tipos de triangulação privilegiados foram os de fontes de evidência e os de método, sendo raros estudos de caso que tenham realizado triangulação teórica, triangulação de investigadores, ou técnicas de triangulação sistematizadas, como a proposta por Triviños (1987). Foi possível verificar, ainda, relatos inadequados da utilização de triangulação nas pesquisas ao não identificarem como a triangulação foi feita, o tipo específico e até mesmo mostrando a adoção de 
procedimentos metodológicos inconsistentes com os propostos pelo autor que se citava.

Como consideração final deste trabalho, reafirma-se a importância da utilização da triangulação, em suas diferentes formas, como estratégia metodológica para aumentar a validade de confiabilidade em investigações de caráter qualitativo, tais como estudos de caso realizados na área de Administração e, além de sua utilização, reforça-se a necessidade de que a triangulação seja relatada de forma transparente e coerente nas versões finais das pesquisas, para tanto, revelando os procedimentos adotados quanto à realização do que foi considerado como triangulação, assim, permitindo ao leitor acessar e julgar tal validade e confiabilidade. 


\section{WÜNSCH TAKAHASHI}

\section{REFERÊNCIAS}

ANGROSINO, M. Etnografia e Observação Participante. Porto Alegre: Artmed, 2009. 15-34 ISBN 9788536321387.

BLUHM, D. J. et al. Qualitative Research in Management : A Decade of Progress. Journal of Management Studies, Dec. 2011.

CORAIOLA, D. M. et al. Estudo de Caso. In: TAKAHASHI, A. R. W. (Ed.). Pesquisa Qualitativa em Administração: Fundamentos, Métodos e Usos no Brasil. São Paulo: Atlas, 2013.

CRESWELL, J. Projeto de pesquisa métodos qualitativo, quantitativo e misto. Porto Alegre: Artmed, 2007.

DENZIN, N.; LINCOLN, Y.; NETZ, S. O planejamento da pesquisa qualitativa teorias e abordagens. Porto Alegre: Artmed, 2007.

EISENHARDT, K. M. Building Theories From Case Study Research Building Theories from Case Study Research. Academy of Management Review, v. 14, n. 4, p. 532-550, 1989.

FLICK, U. Qualidade na Pesquisa Qualitativa. Porto Alegre: Artmed, 2009.

GIBBERT, M.; RUIGROK, W. The "What" and 'How" of Case Study Rigor: Three Strategies Based on Published Work. Organizational Research Methods, 2010.

GIBBERT, M.; RUIGROK, W.; WICKI, B. What Passes as a Rigorous Case Study? Strategic Management Journal, v. 29, p. 1465-1474, 2008.

LINCOLN, Y. S.; GUBA, E. G. Naturalistic Inquiry. Newbury Park, CA, Sage Publications, 1985.

MERRIAM, S. B. Qualitative Research and Case Study Applications in Education: Revised and Expanded from Case Study Research in Education. San Francisco: Jossey-Bass Publishers, 1998.

SANDBERG, J. How Do We Justify Knowledge Produced Within Interpretive Approaches ? Organizational Research Methods, v. 8, n. 1, p. 41-68, 2005. ISSN 1094428104272.

TRIVIÑOS, A. N. S. Introdução à Pesquisa em Ciências Sociais - A pesquisa qualitativa em educação. São Paulo: Atlas, 1987. ISBN 8522402736.

YIN, R. Estudo de caso: planejamento e métodos. Porto Alegre: Bookman, 2010.

Qualitative research from start to finish. New York: Guilford Press, 2011.

ZAPPELLINI, M. B.; FEUERSCHÜTTE, S. G. O Uso Da Triangulação Na Pesquisa Científica Brasileira Em Administração. Administração: Ensino e Pesquisa, v. 16, n. 2, p. 241-241, 2015. 


\section{DADOS DOS AUTORES}

\section{CAMILA BRUNING ${ }^{\star}$ camila.bruning@gmail.com}

Doutora em Administração pela UFPR

Instituição de vinculação: Universidade Federal do Paraná

Curitiba/PR - Brasil

Áreas de interesse em pesquisa: Estudos Organizacionais Críticos, Psicologia

Organizacional, Psicologia do Trabalho, Psicologia Crítica.

* Departamento de Psicologia/UFPR - Praça Santos Andrade, 50 (Ala Alfredo Buffren), Sala 215

- $2^{\circ}$ andar $80060.240 \quad$ Curitiba/PR

\section{LUCIANA GODRI lugodri@gmail.com}

\section{Mestre em Administração pela Universidade Positivo}

Instituição de vinculação: Doutoranda pela Pontifícia Universidade Católica do Paraná Curitiba/PR - Brasil

Áreas de interesse em pesquisa: Estudos Organizacionais, Construcionismo Social, Institucionalismo Organizacional, Sociologia do Direito, Prática Estratégica.

\section{ADRIANA ROSELI WÜNSCH TAKAHASHI adrianarwt@gmail.com} Doutora em Administração pela USP

Instituição de vinculação: Universidade Federal do Paraná

Curitiba/PR - Brasil

Áreas de interesse em pesquisa: Capacidades dinâmicas, aprendizagem e conhecimento organizacional, competências organizacionais e pesquisa qualitativa. 